\title{
The control of abstinence in the treatment of alcohol dependence: the use of acamprosate in relapse prevention
}

\author{
Fabio Venturella, ${ }^{1}$ Anna Asaro, ${ }^{1}$ Guido Faillace, ${ }^{2}$ Gianpaolo Spinnato, ${ }^{3}$ Danila Di Majo, ${ }^{4}$ \\ Maurizio La Guardia, ${ }^{4}$ Marco Giammanco, ${ }^{4}$ Stefania Aiello ${ }^{5}$ \\ ${ }^{1}$ Department of Sciences for Health Promotion G. D'Alessandro, University of Palermo; \\ ${ }^{2}$ Alcamo Drug Addiction Service, Alcamo; ${ }^{3}$ Psichiatry Institute, Local Health Service 2, Palermo; \\ ${ }^{4}$ Department of Law, Society and Sports Studies, University of Palermo; ${ }^{5}$ Department of Molecular \\ and Biomolecular Sciences and Technologies, University of Palermo, Italy
}

\begin{abstract}
Treatment with acamprosate is a valid tool to complement psychotherapy as it does not cause addiction, abuse or withdrawal of its suspension and does not interfere with other medications that patients often alcoholics must take. To evaluate the effectiveness, our study evaluated the effects of Acamprosate compared to $\gamma$-hydroxybutyrate in clinical-physiological and social health in a way indicator of a possible therapeutic success in terms of abstinence from alcohol and social reintegration. A total of 36 patients were observed, of which 5, 4 men and 1 woman at the Drug Addiction Service (Servizio Tossicodipendenze, Ser.T) Alcamo, and 31, 21 men and 10 women at the Ser.T of Palermo. As regards the craving, during the period of treatment with acamprosate, there has been a change, in the sense of reduction, of craving for alcohol: if before therapy was in $68 \%$ of cases, medium-high, it becomes after 3-4 months of therapy in low-nil in $89 \%$ of patients observed. It has been recorded that, after 3-4 months after receiving acamprosate, the clinical picture of the patient is greatly improved by referring to biological markers. In particular, the strong point seems to be the ability for the user to experience a new sense of normalcy and to remove the desire for significant periods of alcohol.
\end{abstract}

\section{Introduction}

Alcoholism can also deal with drug treatments. This is the message that emerges from the press conference of presentation of Campral ${ }^{\circledR}$, \footnotetext{
90127 Palermo, Italy.

Tel. +39.091.6553614 - Fax: +39.091 .6553641 .

E-mail: fabio.venturella@unipa.it

Key words: alcoholism, acamprosate, relapse.

(C) Copyright F. Venturella et al., 2014

Licensee PAGEPress, Italy

Journal of Biological Research 2014; 87:2142

doi:10.4081/jbr.2014.2142
}

Correspondence: Fabio Venturella, Department of Sciences for Health Promotion G. D’Alessandro, University of Palermo, via del Vespro 133,

This article is distributed under the terms of the Creative Commons Attribution Noncommercial License (by-nc 3.0) which permits any noncommercial use, distribution, and reproduction in any medium, provided the original author(s) and source are credited. trade name of acamprosate, a specifically neuromodulator indicated in the maintenance of abstinence in alcohol-dependent patients. ${ }^{1}$ Alcoholism is a disease characterized by: craving, loss of control, tolerance and physical dependence. ${ }^{2}$ Aim of the study was to clinically prove efficacy, influence and usefulness of acamprosate in reducing craving during abstinence in alcoholics. To evaluate the real effectiveness, our study evaluated the effects of acamprosate, compared to $\gamma$-hydroxybutyrate (GHB). Through an antagonistic action of glutamatergic hyperactivity that characterizes alcohol abstinence, acamprosate reduces the craving, the incidence and severity of relapses in alcoholic patients. ${ }^{3}$ It is available in tablets of $333 \mathrm{mg}$ and the recommended dosage is 2 tablets 3 times a day. Concomitant use of alcohol has no effect on the acamprosate pharmacokinetics, suggesting that patients can continue treatment even during relapse periods without health risks. ${ }^{4}$ There are no signs of tolerance, dependence, withdrawal syndrome; the percentages of relapses after stopping treatment are very low. Unlike other anticraving drugs, acamprosate presents an over time growing effectiveness.

\section{Materials and Methods}

The structures that have joined the study were Drug Addiction Service (Servizio Tossicodipendenze, Ser.T) of Alcamo and the Palermo's district 11. In total, from May 2011 to March 201236 patients were observed, of which 5 ( 4 men and 1 woman) at the Sert of Alcamo and 31, including 21 men and 10 women at the Ser.T of Palermo. The patients for whom we have adopted the treatment with acamprosat are $22.2 \%$. General data such as sex, age, education, employment and clinical data and the possible positive and/or the pattern of diseases related to addiction have been reviewed. Of the sample, $69.5 \%$ were male, while women represented only $30.5 \%$. Of the sample, $52.8 \%$ were aged between 35 and 50 years, $30.5 \%$ over 50 years, while only $16.7 \%$ of the sample between $25-35$ years. Patients already attending the Ser.T are treated with GHB and are subjected for the first time treated with acamprosate, and have different expectations of the drug: succeed to terminate therapy with GHB, stop the use of alcohol, overcome the withdrawal symptoms and cease the use of drugs. Of patients, $58.3 \%$ revealed frequent and intense relapses during treatment with GHB. Before starting treatment with acamprosate all patients underwent medical and toxicological assessment (clinical examination, medical history and general toxicology, urinalysis, blood alcohol test, psychological examination) and were informed about the acamprosate characteristics and action mechanism. 


\section{Results}

From data obtained by medical records of patients already being treated with GHB peaks of aspartate amino-transferase (AST) of 920 $\mathrm{UI} / \mathrm{L}$, alanine transaminase (ALT) $670 \mathrm{UI} / \mathrm{L}$ and $\gamma$-glutamyltransferase (GGT) $1700 \mathrm{UI} / \mathrm{L}$ were encountered. Weekly, through medical examination and an interview with a psychologist, we evaluated the clinical course of the patient, the patient's response to acamprosate, any impact resulting from the reduction of the use of alcohol by the use of GHB and possible side effects. Permanently suspended the use of GHB, the patient continued treatment with only acamprosate: to this point we have carried out new surveys, recording the data obtained after 3-4 months from suspension of GHB and after the end of therapy. The percentage of relapse during treatment with $\mathrm{GHB}$ was $55.6 \%$, while with acamprosate only $13 \%$. According to the latest findings obtained after 6-8 months after treatment with acamprosate, the marker's values are returned in standard: the average of AST was reduced from 77 to 17 $\mathrm{UI} / \mathrm{L}$, those of ALT 43.3 to $21 \mathrm{UI} / \mathrm{L}$, and that of GGT from 356.8 to $61 \mathrm{UI} / \mathrm{L}$. Almost all the sample believed acamprosate subjectively more effective than GHB (obviously those who have experienced the two drugs).

The reason for the preference of acamprosate to GHB is given by the lesser dependence towards the drug and the alcohol decreased desire. The majority of patients experience a more relaxed, understood as a reduction in anxiety, nervousness as well as increased ability to concentrate. The alcohol craving during treatment with acamprosate decreased in subjects clearly treated: if before treatment it was medium-high in $68 \%$ of cases, it became a low-void $3-4$ months in $89 \%$ of patients observed. The success rate of treatment with acamprosate was very high at $86 \%$, but should be considered an abandonment of therapy (14\%) caused by the appearance of side effects such as bloating medium-high (1 case), ulcerative (1 case), sexual dysfunction (2 cases), and psychiatric disorders (that may already exist).

\section{Discussion}

Acamprosate is a drug that acts on the glutamatergic system; it seems useful not only in the control of mood but also in improving the withdrawal symptoms and decrease the number of relapses. Through an antagonistic action of glutamatergic hyperactivity that characterizes abstinence from alcohol, acamprosate reduces the craving, the incidence and severity of relapse in alcoholics subjects. Acamprosate is also useful to reduce or cancel the appearance of acquired stimuli leading to the alcohol dependence in time: in this sense inhibition of glutamatergic transmission by the acamprosate is able to reduce the severity of abstinence's syndrome. On the other hand, its use must have a sufficient duration to allow neuronal excitability normalization in the most enduring possible: the treatment, in fact, is recommended for one year. In any case, the use can be continued even in the face of relapses, with the aim to reduce the frequency or severity.

\section{Conclusions}

The hope for the future is that a growing experience by clinicians can facilitate, over time, an optimal selection of suitable patients for treatment with acamprosate.

\section{References}

1. Mason BJ, Lehert P. Effect of acamprosate on protracted withdrawal symptoms: anxiety, dysphoria and insomnia. 47th Annual Meeting of the College of Neuropsychopharmacology, 7-11-2008, Scottsdale, AZ, USA.

2. Mason BJ, Heyser CJ. Acamprosate: a prototypic neuromodulator in the treatment of alcohol dependence. CNS Neurol Disord Drug Targets 2010;9:23-32.

3. Ootemen W, Naassila M, Koeter MW, et al. Predicting the effect of naltrexone and acamprosato in alcohol-dependent patients using genetic indicators. Addict Biol 2009;14:328-37.

4. Pilling S, Yesufu-Udechuku A, Taylor C, Drummond C. Diagnosis, assessment and management of harmful drinking and alcohol dependence: summary of NICE guidance. Brit Med J 2011; 342:d700. 